\title{
Tangence
}

\section{La lecture littéraire au secondaire : le point de vue de la didactique}

\section{Michel Thérien}

Numéro 36, mai 1992

La lecture littéraire

URI : https://id.erudit.org/iderudit/025713ar

DOI : https://doi.org/10.7202/025713ar

Aller au sommaire du numéro

Éditeur(s)

Tangence

ISSN

0226-9554 (imprimé)

1710-0305 (numérique)

Découvrir la revue

Citer cet article

Thérien, M. (1992). La lecture littéraire au secondaire : le point de vue de la didactique. Tangence, (36), 75-84. https://doi.org/10.7202/025713ar d'utilisation que vous pouvez consulter en ligne.

https://apropos.erudit.org/fr/usagers/politique-dutilisation/ 


\section{La lecture littéraire au secondaire: le point de vue de la didactique}

\section{Michel Thérien}

Périodiquement, pour ne pas dire continuellement, les médias électroniques et la presse écrite portent les mêmes constats sur la situation du français dans les écoles: les élèves ne lisent plus, ils ne savent plus écrire. Sans analyser longuement les présupposés de telles observations, on conviendra qu'elles permettent d'espérer qu'il existait un temps où les élèves lisaient plus et écrivaient mieux. Les comparaisons à trente ans de distance sont difficiles à faire tant les situations ont changé, mais à qui ne les aurait pas lues, je suggère Les insolences du frère Untel pour apprécier la situation du français dans les écoles publiques du Québec d'avant la réforme.Plus récemment, un nouveau reproche était fait à l'école: on ne lit plus les grands auteurs. Il est vrai qu'à part quelques extraits dans les recueils de morceaux choisis, les grands auteurs ne sont guère présents. Je me demande malgré tout si on ne lit pas actuellement davantage d'œuvres littéraires au secondaire que par le passé: je ne parle évidemment pas des dernières années du secondaire classique où l'on étudiait la littérature et qui correspondent maintenant au niveau collégial. C'est dans ce contexte que j'aborderai la lecture littéraire au secondaire, d'un point de vue didactique. Mon exposé comprendra trois parties: dans un premier temps, je présenterai les grandes orientations de la pratique du discours littéraire proposées par le programme de français et la recherche; puis, à partir de la théorie de Bakhtine, je montrerai dans quel sens on peut enrichir la notion de lecture littéraire au secondaire; enfin plus brièvement, je regarderai comment une poétique à la Bachelard et une herméneutique à la Ricœur complètent nos propositions pour une nouvelle conception de la lecture littéraire au secondaire.

\section{La pratique du discours littéraire proposée par le programme de français et la recherche}

À ses deux grands objectifs, qu'il partage avec celui du primaire (compréhension et production de discours), le programme 
du secondaire ${ }^{1}$ en ajoute un troisième pour la classe de français, soit que l'élève se situe par rapport aux valeurs socio-culturelles véhiculées par les discours. Dans une large mesure et sans être exclusif, cet objectif est souvent associé au discours littéraire. Il s'agit cependant d'un nouvel objectif, cela n'est pas une exception, il en va de même pour tous les autres objectifs du programme.

J'ai utilisé le terme de "discours littéraire": jamais pourtant il n'est fait référence à ce terme ni à celui de littérature. Le programme propose plutôt les notions de discours narratif, de discours descriptif et de discours poétique. De la même façon, ni dans les guides, ni dans le programme, un cadre théorique de référence n'est présenté. Des révisions sont en cours, qui proposent une autre typologie et une autre terminologie, et il n'est toujours pas question directement de littérature mais plutôt de textes à structure narrative, explicative, descriptive, argumentative, de résolution de problèmes, etc.

Le programme de secondaire propose deux entrées différentes au texte: la situation de communication et le fonctionnement de la langue et des discours. La première, qu'on pourrait appeler pragmatique et qui est centrée sur les partenaires de l'acte de communication, ignore le discours littéraire, qui n'apparaît que dans la seconde entrée, le fonctionnement de la langue et des discours. Sans entrer dans les détails, ni dans les querelles d'école ou de terminologie, on pourrait dire qu'il s'agit d'une approche structurale et sémiotique, les deux grandes références implicites étant Gérard Genette et A. J. Greimas. L'essentiel des contenus d'apprentissage proposés porte sur la forme et sur la reconnaissance de caractéristiques génériques, principalement narratologiques. De la $1^{\text {re }}$ à la $5^{\mathrm{e}}$ secondaire, les contenus s'élargissent et les types de discours se complexifient (récit, conte, nouvelle, roman, par exemple), mais la démarche reste toujours la même et la perspective globale inchangée. On insiste cependant sur la lecture d'œuvres intégrales, rejetant ce que l'on pourrait appeler la pédagogie des extraits.

Parallèlement à cette vision, en recherche principalement, s'est développé tout un ensemble d'études sur la lecture, avant

1 Ministère de l'Éducation du Québec, Programme d'études, Français langue maternelle, $1^{r *}, 2^{*}, 3^{*}, 4^{*}, 5^{*}$ secondaire, Québec, Direction de la formation générale, 1980 . 
tout inspirées de la psychologie cognitive et portant presque exclusivement sur des questions de forme, les notions de schéma, de script, de scénario ou de frame occupant les devants de la scène. Depuis une vingtaine d'années, on est passé des questions de méthodes à des questions de processus, où apparaît la notion centrale de compréhension. Un intérêt commence à poindre cependant pour les stratégies d'apprentissage que les maîtres devraient employer auprès des élèves. En ce qui concerne les processus de compréhension, le récit - généralement très court s'est imposé, et il n'est pas exagéré de dire que la spécificité du littéraire n'y a pas trouvé son compte. Les travaux de ce que j'appellerai l'École de Chicoutimi (autour de Ghislain Bourque) font exception à cette critique puisqu'ils accordent une grande importance à la matérialité discursive, intégrant lecture et écriture. Quant aux stratégies d'apprentissage, en pleine émergence, les discours non littéraires s'y imposent, laissant une faible place au littéraire.

Malgré leurs défauts, les typologies ministérielles, qui ne sont pas les mêmes au primaire et au secondaire, ont l'immense mérite de reconnaître, malgré tout, l'intérêt du littéraire, puisque celui-ci est intégré aux programmes: le poème est étudié à côté de la lettre d'opinion et la nouvelle littéraire à côté de la nouvelle journalistique. On suggère justement les comparaisons entre différents types de discours afin de reconnaître leurs caractères génériques.

Cette réduction de la place du texte littéraire au profit des autres discours sociaux est un reproche fréquent. Fini le temps béni où tous les extraits lus en classe, tous les exemples de grammaire, tous les exercices à faire se référaient aux grands auteurs. On remarquera que c'est encore vrai, dans une large mesure, pour les exemples des dictionnaires. Pour ma part, c'est un reproche que je récuse, principalement pour deux raisons: d'une part, en ne laissant aucune place aux autres discours, l'école véhiculait une vision très limitée des usages de la langue et ne rendait pas nécessairement justice au littéraire; d'autre part, avec la démocratisation de l'enseignement, l'école s'est ouverte à une clientèle très différente de celle qui fréquentait naguère l'école secondaire et les besoins langagiers de l'ensemble de la jeunesse dépassent largement la seule fréquentation des textes littéraires. Il faut comprendre que la maîtrise de la langue, en situation de réception et de production, est un important facteur 
de pouvoir social et, qu'à ce titre, l'école démocratique doit permettre à l'ensemble de la jeunesse de la posséder et notamment de rendre les jeunes aptes à la pratique des différents discours en usage dans la société.

Il est souhaitable cependant que le corpus théorique de référence sur la lecture littéraire au secondaire soit élargi, qu'elle ne soit pas limitée à une approche de type structural ou narratologique, bref, qu'une pluralité d'approches vienne en enrichir la pratique.

\section{Bakhtine et la lecture littéraire au secondaire}

Depuis plusieurs années, cette question de la lecture littéraire au secondaire me préoccupe, non pas que je trouve qu'on devrait enseigner davantage la littérature mais qu'on doive l'enseigner autrement. Plusieurs raisons justifient à la fois ce constat et le choix que je fais de Bakhtine. D'une part, l'âge des élèves: l'adolescence est l'âge des grandes questions et une approche qui ne s'intéresse qu'aux problèmes de forme indépendamment des contenus fait fi du développement cognitif et des besoins affectifs des jeunes. À quoi peut-il servir de lire à quinze ans La mort du petit cheval ou Le canard de bois en ne s'intéressant qu'à la logique des actions? D'autre part, le programme, accordant une large place aux valeurs socio-culturelles (c'est le troisième objectif, il me semble tout à fait inapproprié de s'en tenir à des questions de forme. À cet égard, l'approche bakhtinienne, fondamentalement axiologique, m'apparait propice à cette lecture critique des systèmes de valeur représentés dans le texte littéraire. Enfin, et cet aspect me semble fondamental, la composition pluriethnique du Québec d'aujourd'hui et de demain - cela est vrai pour les écoles montréalaises mais concerne également l'ensemble du système scolaire - remet en cause l'enseignement, particulièrement l'enseignement du français, qui doit faire une large place à la dimension culturelle de l'apprentissage. Or, la lecture littéraire se situe au centre de cette dimension. Les concepts bakhtiniens proposés sont principalement empruntés à Esthétique et théorie du roman ${ }^{2}$ et ils concernent avant tout la prose romanesque. Deux de mes étudiantes, l'une au niveau de la

Mikhaïl Bakhtine, Esthétique et théorie du roman, Paris, Gallimard, 1978. 
maitrise ${ }^{3}$, l'autre au niveau du doctorat ${ }^{4}$, ont montré l'intérêt didactique de cette approche pour la lecture littéraire au secondaire.

Le premier concept sur lequel je voudrais attirer l'attention est celui de polyphonie, incluant à la fois la pluralité des genres et la structuration sociale des discours. Je n'insisterai guère sur la pluralité des genres, encore qu'elle implique une compétence complexe, puisqu'elle appelle la reconnaissance de plusieurs formes de discours, la lettre, le sermon, le discours didactique, par exemple, et qu'elle renvoie souvent à la réécriture parodique. Une telle approche implique un travail sur les discours premiers et pose aux maîtres le délicat problème des antécédents et des référents du texte littéraire, de même que celui des compétences de lecture. La structuration sociale des discours, est, quant à elle, fondamentale et se rattache intimement à l'objectif d'appropriation des valeurs socio-culturelles. Sociolinguiste avant le temps, Bakhtine s'intéresse non pas tant à la variété linguistique qu'à ce qu'il appelle la structuration sémantique et axiologique des discours. Il affirme même que ce qui caractérise le personnage du roman, ce ne sont pas ses actions, mais ses paroles. $\grave{A}$ ce titre, écrit-il, tout personnage de roman est un idéologue. Les étudiants, pourtant, ont beaucoup de difficultés à voir dans les confrontations de l'univers romanesque représenté autre chose que des conflits psychologiques, des conflits de caractère, même s'ils sont prêts par ailleurs à affirmer qu'il s'agit d'êtres de papier.

Intimement lié au concept de polyphonie, le concept de bivocalité me semble aussi fondamental, pour orchestrer à la fois les différents discours des personnages, le discours du narrateur de même que le discours de l'auteur. Je sais que le rôle imparti à l'auteur est particulièrement problématique, surtout en narratologie, où il est gommé. Il semble être très près de celui du narrateur, mais il n'en reste pas moins qu'un grand nombre d'énoncés conjuguent à la fois le discours de celui qui parle et de celui dont on parle. Si l'on ne travaille pas ce genre de problème avec les élèves, on risque d'aplatir considérablement la polyphonie romanesque et

3 F. Coulombe, L'apport de Bakbtine à la didactique du roman, mémoire de maîtrise, Université de Montréal, Faculté des études supérieures, 1986.

4 J. Jean, Élaboration d'un guide pédagogique pour une lecture bakbtinienne et interactionniste du roman, thèse de doctorat, Université de Montréal, Faculté des études supérieures, 1989. 
80

de méconnâtre la nature idéologique et bivocale du roman. C'est un autre aspect extrêmement important de la compétence littéraire. Enfin, la polyphonie implique l'intertextualité et l'interdiscursivité. Sans avoir l'ambition de faire avec les élèves ce que Marc Angenot a fait avec $1889^{5}$, il n'en reste pas moins que l'œuvre littéraire est, dans une large mesure, marquée par le contexte discursif et social qui l'a vue naître et que des éléments importants de sa compréhension ont à voir avec la série dans laquelle elle s'inscrit, qu'elle complète et explique en quelque sorte. Sans vouloir retourner à l'histoire littéraire, j'affirme, pour ma part, que la compétence littéraire inclut nécessairement des connaissances historiques et contextuelles. À ce titre, la notion de corpus d'œuvres à lire devrait être réintroduite dans le programme. Je sais que c'est une notion piégée, mais elle m'apparaît fondamentale pour comprendre le littéraire. Dans le contexte qui est le nôtre au Québec aujourd'hui, je n'hésite pas à dire que ce corpus doit être essentiellement québécois, tout en faisant appel aux œuvres de la culture immigrée et en s'ouvrant à la francophonie (des textes de la France certes, mais aussi de l'Afrique et des Antilles). De la même façon, ce corpus doit s'ouvrir aux œuvres cinématographiques ou télédiffusées car la compétence médiatique doit être considérée comme une autre dimension de la compétence culturelle, intimement liée à la compétence littéraire ${ }^{6}$.

Le second concept sur lequel je voudrais insister est celui de chronotope, non pas tant le chronotope romanesque, encore qu'il constitue une approche intéressante de ce que Bakhtine appelle la concrétisation de l'axiologie de l'œuvre et de l'image de l'homme et du monde, mais ce qu'il appelle le chronotope de la lecture ${ }^{7}$. En pédagogie de la littérature, la responsabilité du maître est énorme, notamment avec des adolescents. Dans la plupart des cas, le premier contact avec une œuvre se fait en salle de classe et peut être déterminant pour son appréciation. Le chronotope de la

5 Marc Angenot, 1889. Un état du discours social, Longueuil, Le Préambule, 1989.

6 Cette compétence médiatique fait partie des programmes de francais en Ontario (ministère de l'Éducation de l'Ontario, La compétence médiatique, Cycles intermédiaire et supérieur, Toronto, Ministère de l'Éducation, 1989).

7 Celui-ci est étudié par M. Szegedy-Maszak dans * Le texte comme structure et construction", in M. Angenot, J. Bessière, D. Fokkema et E. Kushner, éd., Théorie littéraire, Paris, PUF, 1989, p. 183-218. 
lecture reconnaît à chaque lecture, située dans un temps et un espace bien précis, une recréation unique. Autant la notion de série et de contexte est fondamentale, autant celle de chronotope de la lecture impose au maitre une concentration importante sur le sujet lecteur, sur ses horizons d'attente et ses capacités de réception, bref, sur ce que l'on pourrait appeler une pédagogie différenciée. En ce sens, les théories interactionnistes de la lecture qui postulent que le sens se construit par l'interaction texte-lecteur me semblent satisfaisantes, dans la mesure où c'est à partir de l'objet-texte que se construit le sens et où tout n'est pas laissé au caprice du lecteur. Cet aspect est assez bien compris par les enseignants et l'on peut dire que la notion analogue de lecture plurielle en littérature a fait son chemin dans les classes de français.

Enfin, la notion de représentation et celle de carnavalisation attirent l'attention sur la recréation de l'œuvre littéraire, sur sa dimension critique et utopique. Les différentes formes de comique, ironie, parodie, satire, les figures du sot et du fripon dont Bakhtine étudie les avatars à travers la production littéraire me semblent particulièrement inspirantes pour donner à lire aux élèves une autre dimension essentielle de l'œuvre littéraire. Intimement liés à l'axiologie de l'œuvre et à sa polyphonie, ces procédés font entrer de plain-pied dans la compréhension de l'œuvre et sont partie intégrante de la compétence littéraire. De nombreuses études ont porté sur la carnavalisation dans la littérature québécoise et il n'est pas exagéré de dire que ce concept facilite l'approche de la prose africaine et caraibe.

Deux mises en garde doivent être faites. Appliquées mécaniquement et systématiquement à toute œuvre romanesque, même graduellement comme le programme le fait pour les outils de la narratologie, ces notions ne garantissent pas ipso facto une lecture littéraire intelligente. En fait, idéalement, chaque œuvre littéraire devrait susciter son appareil critique, d'autant plus que nous avons affaire à des adolescents en processus d'appropriation de stratégies de lecture. Ce qu'on doit surtout retenir, c'est qu'il ne faut pas enfermer l'œuvre dans une seule lecture et qu'il faut se méfier des pédagogisations rapides, définitives et réifiantes. D'un autre côté, l'appropriation de ces notions demande du temps et de la formation: au terme de plusieurs années d'un séminaire de maîtrise sur cette approche, je puis dire qu'elle peut se pratiquer par les enseignants et que leur savoir leur permet d'en proposer des adaptations et des démarches appropriées aux capacités et aux 
besoins de développement de leurs élèves ${ }^{8}$. L'approche bakhtinienne constitue un complément qui s'articule harmonieusement à l'approche narratologique et qui pourrait contribuer à l'atteinte du troisième objectif du programme, à savoir l'appropriation critique des valeurs.

Voilà, à partir de Bakhtine, quelques pistes intéressantes pour enrichir la lecture littéraire au secondaire et contribuer à une meilleure compréhension des dimensions de cette compétence polymorphe, essentielle au développement personnel et social. Pour terminer, amorçons une réflexion sur l'imaginaire et l'herméneutique.

\section{Poétique et herméneutique}

Dans une large mesure, la pratique de la littérature repose, autant en réception qu'en production, sinon sur une frustration, un manque, une incomplétude que l'œuvre contribue à combler. Si l'adolescent lit des récits d'aventure ou des romans d'amour, c'est, entre autres choses, parce qu'il ne peut les vivre dans la réalité. De même que Balzac pouvait payer ses dettes par procuration en faisant de César Birotteau le martyr de la probité commerciale, de même il faut permettre aux jeunes de vivre dans l'imaginaire des rêves qu'ils ne peuvent vivre dans la réalité. Comme l'écrit Marthe Robert $^{9}$, la littérature est comme le rêve de l'humanité où tous les fantasmes sont déposés et transposés de telle sorte que chacun puisse les vivre par procuration et sans culpabilité.

Comme le dit Bachelard, il faut donner aux élèves le droit de rêver, il faut redonner à l'élève la "culture des songes", selon la belle expression de Bruno Duborgel, empruntée à son livre, Imaginaire et pédagogie:

Éduquer le sujet de rationalité, déployer l'imaginaire de chacun et au fil de ces développements inverses, faire naître le sujet lui-même comme "pluralisme cohérent", tel est le programme immense à penser, telle est la tâche générale de l'École, tant il est vrai "que l'homme diurne et l'homme nocturne" constituent

8 M. Thérien, * La lecture du roman au secondaire *, in $M$. Thérien et G. Fortier, éd., Didactique de la lecture au secondaire, Montréal, Éditions Ville-Marie, 1985, p. 221-242.

9 Marthe Robert, La tyrannie de l'imprimé, Livres de lectures III, Paris, Grasset et Fasquelle,1984. 
la double base d'une anthropologie complète, "c'est-à-dire d'une sagesse ou écologie de la psyché ${ }^{1}{ }^{10}$

Les enseignants connaissent la stylistique et l'on retrouve fréquemment des exercices sur l'identification des figures, notamment la métaphore et la comparaison. On comprendra que le travail sur les images dépasse considérablement l'identification et peut ouvrir à l'inconscient du texte. Même en prose, l'approche bachelardienne des images constituerait un supplément très enrichissant à l'approche bakhtinienne; elle me semble un passage obligé en poésie. Elle attire à la fois l'attention sur la matérialité même du texte, la constitution d'importantes isotopies discursives et l'investissement affectif du lecteur. Gommer cette dimension de la lecture littéraire, même en prose, serait méconnaître une des facettes fondamentales du texte littéraire.

Enfin, l'œuvre littéraire transmet une somme incalculable de réflexions, de pensées, d'expérience, de sagesse, en attente d'être partagées, d'être critiquées, dans une réception dialogique. Paul Ricœur écrit à ce propos: "Il n'est pas de compréhension de soi qui ne soit médiatisée par des signes, des symboles et des textes; la compréhension de soi coïncide à titre ultime avec l'interprétation appliquée à ces termes médiateurs" 11 . Le concept de monde représenté que Ricour applique au texte littéraire et dont il dit qu'il contient peut-être la meilleure part de l'héritage culturel me semble un concept fondateur de la lecture littéraire au secondaire. Priver les jeunes de cette ouverture à l'Autre, par une conception restreinte du texte littéraire, mapparaît être une aberration. L'un des rôles fondamentaux de l'école, c'est le développement et la transmission du patrimoine culturel, et l'un des véhicules privilégiés de son expérience, de ses doutes ou de ses croyances, de ses forces ou de ses faiblesses est la littérature.

\section{Conclusion}

Le programme de français, en reconnaissant la multiplicité des discours, détermine en quelque sorte la spécificité du

10 Bruno Duborgel, *Décoloniser l'imaginaire *, Vision, 37, 1985, p. 23; voir aussi, du même auteur, Imaginaire et pédagogie, Paris, Le sourire qui mord, 1983.

11 Paul Ricœur, Du texte à l'action, Essais d'berméneutique II, Paris, Seuil, coll. "Esprit”, 1986. 
84

littéraire. Si le programme et les enseignants ont bien intégré les acquis de l'approche structurale et narratologique, le moment est venu d'élargir les référents théoriques capables de renouveler la lecture littéraire au secondaire.

Sans être exhaustives, les propositions faites ici et principalement empruntées à Bakhtine, Bachelard et Ricœur favoriseraient l'atteinte du troisième objectif du programme: l'appropriation critique des valeurs, et ouvriraient davantage les pratiques de lecture au contenu, aux images et à l'interprétation des œuvres.

La compétence littéraire est une compétence complexe, mais elle ne doit pas être réservée à une élite, elle doit être la quête de toute la jeunesse, la conquête de Soi et de l'Autre. 